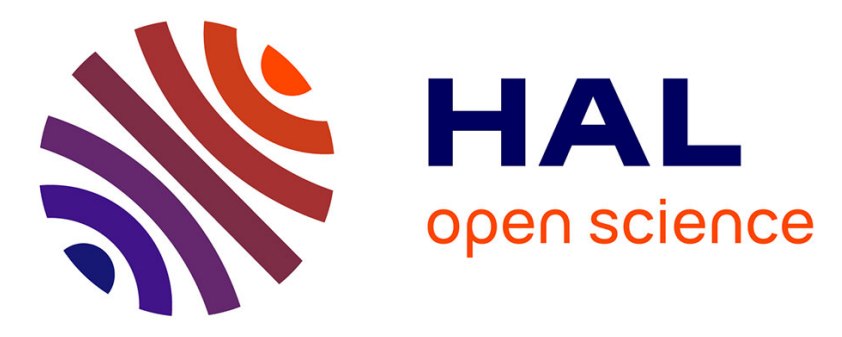

\title{
Density fluctuations of water-glucose mixtures studied by Inelastic Ultra-Violet Scattering
}

\author{
Mariaelena Gallina, Lucia Comez, Stefania Perticaroli, Assunta Morresi, \\ Attilio Cesàro, Ornela de Giacomo, Silvia Di Fonzo, Alessandro Gessini, \\ Claudio Masciovecchio, Luciano Palmieri, et al.
}

\section{To cite this version:}

Mariaelena Gallina, Lucia Comez, Stefania Perticaroli, Assunta Morresi, Attilio Cesàro, et al.. Density fluctuations of water-glucose mixtures studied by Inelastic Ultra-Violet Scattering. Philosophical Magazine, 2008, 88 (33-35), pp.3991-3998. 10.1080/14786430802481903 . hal-00513977

\section{HAL Id: hal-00513977 \\ https://hal.science/hal-00513977}

Submitted on 1 Sep 2010

HAL is a multi-disciplinary open access archive for the deposit and dissemination of scientific research documents, whether they are published or not. The documents may come from teaching and research institutions in France or abroad, or from public or private research centers.
L'archive ouverte pluridisciplinaire $\mathbf{H A L}$, est destinée au dépôt et à la diffusion de documents scientifiques de niveau recherche, publiés ou non, émanant des établissements d'enseignement et de recherche français ou étrangers, des laboratoires publics ou privés. 


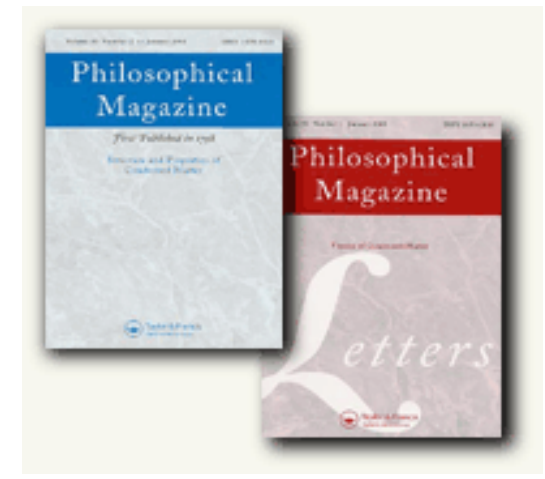

Density fluctuations of water-glucose mixtures studied by Inelastic Ultra-Violet Scattering

\begin{tabular}{|c|c|}
\hline Journal: & Philosophical Magazine \& Philosophical Magazine Letters \\
\hline Manuscript ID: & TPHM-08-May-0163.R1 \\
\hline Journal Selection: & Philosophical Magazine \\
\hline Date Submitted by the Author: & 04-Aug-2008 \\
\hline Complete List of Authors: & $\begin{array}{l}\text { Gallina, Mariaelena; Università di Perugia, Chimica } \\
\text { Comez, Lucia; Università di Perugia, Dipartimento di Fisica; INFM CRS-SOFT, Università } \\
\text { di Roma DLa SapienzaD } \\
\text { Perticaroli, Stefania; Università di Perugia, Chimica } \\
\text { Morresi, Assunta; Università di Perugia, Chimica } \\
\text { Cesàro, Attilio; Università di Trieste, Biochimica, Biofisica e Chimica delle Macromolecole } \\
\text { De Giacomo, Ornela; Università di Trieste, Biochimica, Biofisica e Chimica delle } \\
\text { Macromolecole } \\
\text { Di Fonzo, Silvia; Sincrotrone Trieste } \\
\text { Gessini, Alessandro; Sincrotrone Trieste, Strada Statale } 14 \text { km 163.5, Area Science Park, } \\
\text { I-34012 Trieste, Italy } \\
\text { Masciovecchio, Claudio; Sincrotrone Trieste } \\
\text { Palmieri, Luciano; Università di Perugia, Dipartimento di Fisica } \\
\text { Paolantoni, Marco; Università di Perugia, Chimica } \\
\text { Sassi, Paola; Università di Perugia, Chimica } \\
\text { Scarponi, Filippo; Università di Perugia, Fisica } \\
\text { Fioretto, Daniele; Università di Perugia, Fisica }\end{array}$ \\
\hline Keywords: & biomolecules, glass transition \\
\hline Keywords (user supplied): & Ultraviolet Brillouin Scattering \\
\hline \multicolumn{2}{|c|}{$\begin{array}{l}\text { Note: The following files were submitted by the author for peer review, but cannot be converted to PDF. You must view these } \\
\text { files (e.g. movies) online. }\end{array}$} \\
\hline Gallina et al - edited.doc & \\
\hline
\end{tabular}

\section{今) ScholaroNE Manuscript Central}

